\title{
ON A NEW TREATMENT OF THEOREMS OF FINITENESS*
}

\author{
BY \\ OLIVER EDMUNDS GLENN
}

Propositions relative to the finiteness of algebraic invariant systems can be treated under the theory of invariants of the type which involves the parameters of the transformations $T$ to which a binary form

$$
f_{m}=\left(a_{0}, \cdots, a_{m}\right)\left(x_{1}, x_{2}\right)^{m}
$$

is subjected. The algorithms of this theory are of extensive content. The full discussion of these functions comprehends not only the ordinary binary algebraic invariant theory as one of its phases, but, as was made clear in detail by the present author, in a former paper, $\dagger$ it comprehends, also, the general theory of orthogonal concomitants, of booleans, and of still other invariant systems appertaining to important special sets $\ddagger$ of binary substitutions. It might have been anticipated, I presume, that an invariant theory based upon considerations connected with the poles of the transformations, as the present theory is based, would be likely to be deeply grounded, but, prior to my own publication on this subject, no one seems to have adopted this point of view.

This paper contains a new and very simple proof of Gordan's theorem, and a determination of the complete system of concomitants in the domain $R(1, T, 0)$, of the binary quintic, carrying with it as one special case, for instance, the previously undetermined complete system of orthogonal concomitants of the quintic form.

As far as my knowledge goes, general proofs of the finiteness of orthogonal or of boolean systems have been given by no one, although these were among the first invariants discovered.§ Apparently this was because researchers long took the erroneous view that these theories were fully included in that of simultaneous concomitants of the ordinary formal type. In this paper I prove these theorems for the general order $m$, and show that the systems are given by the finite set of irreducible solutions of a definite linear diophantine equation.

* Presented to the Society, December, 1917, and September, 1918.

$\dagger$ These Transactions, vol. 18 (1917), p. 443.

$\ddagger$ Annals of Mathematics, vol. 20 (1918), p. 125.

$\S$ Boole, Cambridge Mathematical Journal, vol. 3 (1843), p. 1. 
1. Certain fundamental systems of the Quintic

The poles of the general transformation

are the roots of the linear forms

$$
T:\left\{\begin{array}{l}
x_{1}=\alpha_{1} x_{1}^{\prime}+\alpha_{2} x_{2}^{\prime}, \\
x_{2}=\beta_{0} x_{1}^{\prime}+\beta_{1} x_{2}^{\prime},
\end{array}\right.
$$

$$
f_{ \pm 1}=2 \beta_{0} x_{1}+\left(\beta_{1}-\alpha_{1} \pm \Delta\right) x_{2},
$$

the latter being, accordingly, covariants of $T$. When $f_{m}$ is expanded in terms of $f_{ \pm 1}$ as arguments:

$$
f_{m}=\sum_{i=0}^{m}\left(\begin{array}{c}
m \\
i
\end{array}\right) \phi_{m-2 i} f_{+1}^{m-i} f_{-1}^{i},
$$

the coefficients $\phi$ of the expansion are linear invariants of $f_{m}$ under $T$ in the domain $R(1, T, \Delta)$, the notation for this domain indicating polynomials rational in the coefficients of $T$, in

$$
\Delta=\sqrt{\left(\alpha_{1}-\beta_{1}\right)^{2}+4 \alpha_{2} \beta_{0}},
$$

and rational numerically. The invariant relations are

where

and

$$
\rho_{ \pm 1}=\frac{1}{2}\left(\alpha_{1}+\beta_{1} \pm \Delta\right), \quad \rho_{+1} \rho_{-1}=D=\alpha_{1} \beta_{1}-\alpha_{2} \beta_{0}
$$

$$
\phi_{m-2 i}^{\prime}=\rho_{1}^{m-2 i} D^{i} \phi_{m-2 i} \quad(i=0, \cdots, m) .
$$

The invariants $\phi_{m-2 i}$ can be given an explicit representation if a symbolical notation is adopted. Thus

$$
f=a_{x}^{m}=\left(a_{1} x_{1}+a_{2} x_{2}\right)^{m}=b_{x}^{m} .
$$

and the inverse of the transformations

is

$$
f_{ \pm 1}=2 \beta_{0} x_{1}+\left(\beta_{1}-\alpha_{1} \pm \Delta\right) x_{2},
$$

$$
T:\left\{\begin{array}{l}
x_{1}=\left(-4 \beta_{0} \Delta\right)^{-1}\left[\left(\gamma_{1}-\Delta\right) f_{+1}-\left(\gamma_{1}+\Delta\right) f_{-1}\right], \\
x_{2}=\left(-4 \beta_{0} \Delta\right)^{-1}\left[2 \beta_{0}\left(-f_{+1}+f_{-1}\right)\right] \quad\left(\gamma_{1}=\beta_{1}-\alpha_{1}\right) .
\end{array}\right.
$$

Hence the expansion (1) is given by $f=\left(-4 \beta_{0} \Delta\right)^{-m}\left\{\left[\left(\gamma_{1}-\Delta\right) a_{1}-2 \beta_{0} a_{2}\right] f_{+1}+\left[-\left(\gamma_{1}+\Delta\right) a_{1}+2 \beta_{0} a_{2}\right] f_{-1}\right\}^{m}$, that is

$$
\begin{array}{r}
\phi_{m-2 i}=(-1)^{i}\left[\left(\gamma_{1}-\Delta\right) a_{1}-2 \beta_{0} a_{2}\right]^{m-i}\left[\left(\gamma_{1}+\Delta\right) a_{1}\right. \\
\left.-2 \beta_{0} a_{2}\right]^{i}\left(-4 \beta_{0} \Delta\right)^{-m}, \\
(i=0, \cdots, m) .
\end{array}
$$

This notation will be especially useful in the second section of this paper. 
If we differentiate $\phi_{m-2 i}$ according to the operator

$$
a_{1} \frac{\partial}{\partial a_{2}}+2 \beta_{0} \frac{\partial}{\partial \gamma_{1}}-\gamma_{1} \frac{\partial}{\partial \dot{\alpha}_{2}},
$$

the result is found to vanish. Hence, since

$$
a_{1} \frac{\partial}{\partial a_{2}}=a_{1}\left[\frac{\partial}{\partial a_{0}} \frac{\partial a_{0}}{\partial a_{2}}+\frac{\partial}{\partial a_{1}} \frac{\partial a_{1}}{\partial a_{2}}+\cdots+\frac{\partial}{\partial a_{m}} \frac{\partial a_{m}}{\partial a_{2}}\right],
$$

the invariant $\phi_{m-2 i}$ satisfies the differential equation

$$
\left(a_{0} \frac{\partial}{\partial a_{1}}+2 a_{1} \frac{\partial}{\partial a_{2}}+\cdots+m a_{m-1} \frac{\partial}{\partial a_{m}}+2 \beta_{0} \frac{\partial}{\partial \gamma_{1}}-\gamma_{1} \frac{\partial}{\partial \alpha_{2}}\right) \phi_{m-2 i}=0 .
$$

Every invariantive function, rational or irrational, of the coefficients and variables of $f_{m}$, of the coefficients of $T$, and of $\Delta$, is expressible in terms of the $\phi-s$ and of $f_{ \pm 1}$. We have only to employ the inverse of the set of linear equations (in $a_{0}, \cdots, a_{m}$ )

$$
\phi_{m-2 i}=\phi_{m-2 i}\left(a_{0}, \cdots, a_{m}\right) \quad(i=0, \cdots, m) .
$$

In the domain $R(1, T, \Delta)$ a complete system is composed of $\phi_{m-2 i}(i=0$, $\cdots, m), f_{+1}, f_{-1}$.

In the domain $R(1, T, 0)$, rational in the coefficients of $T$, a complete system is in one-to-one correspondence with those invariantive products

$$
P=\prod_{i=0}^{m} \phi_{m-2 i}^{x_{i}} f_{+1}^{\sigma_{1}} f_{-1}^{\sigma_{3}}
$$

for which the exponent of $\rho_{1}$ in the invariant relation

$$
P^{\prime}=\rho_{1}^{a} D^{b} P
$$

is zero, and for this reason complete systems in this domain are given by the complete set of irreducible solutions in positive integers of the linear diophantine equation

$$
a=\prod_{i=0}^{m}(m-2 i) x_{i}-\sigma_{1}+\sigma_{2}=0 .
$$

The particularization of $T$ which changes the systems belonging to the domain $R(1, T, 0)$ into boolean concomitants is given by

$$
\alpha_{1}=\frac{\sin (\omega-\alpha)}{\sin \omega}, \quad \alpha_{2}=\frac{\sin (\omega-\beta)}{\sin \omega}, \quad \beta_{0}=\frac{\sin \alpha}{\sin \omega}, \quad \beta_{1}=\frac{\sin \beta}{\sin \omega} .
$$

When $\omega=\frac{1}{2} \pi$ the latter systems become orthogonal. We have now proved the following

TheOREM. Complete systems in the domain $R(1, T, 0)$, booleans, ortho- 
gonal systems, as well as concomitants of $f_{m}$ under the so-called relativity* transformations

$$
T:\left\{\begin{array}{l}
t=\frac{1}{\sqrt{1-\beta^{2}}}\left(t^{\prime}+\frac{v}{c^{2}} x^{\prime}\right), \\
x=\frac{1}{\sqrt{1-\beta^{2}}}\left(x^{\prime}+v t^{\prime}\right),
\end{array}\right.
$$

are finite, and are given by the finite set of irreducible solutions in positive integers of the equation (6).

In the instance $m=5$, therefore, we have to solve the equation

$$
5 x_{0}+3 x_{1}+x_{2}+\sigma_{2}=\sigma_{1}+x_{3}+3 x_{4}+5 x_{5}
$$

and if an irreducible solution is given by the $\xi$-s in the first row in the table following, another irreducible solution representing the product conjugate to the first product is furnished by the second row:

\begin{tabular}{cccccccc}
$x_{0}$ & $x_{1}$ & $x_{2}$ & $\sigma_{2}$ & $x_{3}$ & $x_{4}$ & $x_{5}$ & $\sigma_{1}$ \\
\hline$\xi_{0}$ & $\xi_{1}$ & $\xi_{2}$ & $s_{2}$ & $\xi_{3}$ & $\xi_{4}$ & $\xi_{5}$ & $s_{1}$ \\
$\xi_{5}$ & $\xi_{4}$ & $\xi_{3}$ & $s_{1}$ & $\xi_{2}$ & $\xi_{1}$ & $\xi_{0}$ & $s_{2}$
\end{tabular}

If the two products $P, Q$ thus found are combined, giving $P \pm Q=\alpha_{ \pm 1}$, then the $\alpha_{ \pm 1}$, deprived of certain irrelevant constant factors, are members of the fundamental system. I have carried out the work of solving (7) and I give in the table below the set of irreducible solutions found. For convenience in reading off the concomitants $P \pm Q$ a solution and its conjugate are juxtaposed under a double notation, as $l_{ \pm 1}$, the concomitants $l_{ \pm 1}$ being, evidently,

$$
l_{ \pm 1}=\phi_{3} \phi_{-1}^{2} f_{+1} \pm \phi_{-3} \phi_{1}^{2} f_{-1} \text {. }
$$

The number of invariants and covariants in this fundamental system including $f_{+1} f_{-1}$, is 42 , of which 15 are pure invariants. The solutions furnishing the latter are placed in the top rows of the table.

A few remarks should perhaps be added as to the method of solving (7). Transposition of terms reduces the equation to

$$
5 x+3 y+z=0,
$$

where

$\left(9_{1}\right) \quad x_{0}-x_{5}=x, \quad x_{1}-x_{4}=y, \quad x_{2}-x_{3}-\sigma_{1}+\sigma_{2}=z$.

The equation (9) is now solved for its irreducible solutions in both positive and negative integers $x, y, z$, and to each of these solutions correspond sets for (7), given by $\left(9_{1}\right)$. The problem is thus divided into a large number of simple mutually exclusive sub-problems.

\footnotetext{
* R. D. Carmichael, Theory of Relativity, p. 44.
} 


\begin{tabular}{|c|c|c|c|c|c|c|c|c|}
\hline & $x_{0}$ & $x_{1}$ & $x_{2}$ & $x_{3}$ & $x_{4}$ & $x_{5}$ & $\sigma_{1}$ & $\sigma_{2}$ \\
\hline$a \ldots \ldots$ & 1 & & & & & 1 & & \\
\hline$b \ldots \ldots$ & & 1 & & & 1 & & & \\
\hline$c \ldots \ldots$ & & & 1 & 1 & & & & \\
\hline$d_{ \pm 1} \ldots\{$ & & 1 & 3 & 3 & 1 & & & \\
\hline$e_{ \pm 1} \ldots$ & 1 & & 5 & 5 & & 1 & & \\
\hline$g_{ \pm 1}$ & 1 & 1 & 2 & 2 & 1 & 1 & & \\
\hline$h_{ \pm 1} \ldots$ & 1 & 2 & 1 & 1 & 2 & 1 & & \\
\hline$i_{ \pm 1 \ldots} \ldots$ & 3 & 5 & & & 5 & 3 & & \\
\hline$j_{ \pm 1} \ldots$ & 2 & 3 & 1 & 1 & 3 & 2 & & \\
\hline$k_{ \pm 1}$. & & & 1 & 1 & & & 1 & 1 \\
\hline$l_{ \pm 1 \ldots}$ & & 1 & 2 & 2 & 1 & & 1 & 1 \\
\hline$m_{ \pm 1}$ & & 1 & 1 & 1 & 1 & & 2 & 2 \\
\hline$n_{ \pm 1}$. & & 1 & & & 1 & & 3 & 3 \\
\hline$o_{ \pm 1} \ldots$ & 1 & & 4 & 4 & & 1 & 1 & 1 \\
\hline$p_{ \pm 1}$ & 1 & & 3 & 3 & & 1 & 2 & 2 \\
\hline$q \pm 1 \ldots$ & 1 & & 2 & 2 & & 1 & 3 & 3 \\
\hline$r_{ \pm 1} \ldots$ & 1 & & 1 & 1 & & 1 & 4 & 4 \\
\hline$s_{ \pm 1}$ & 1 & & & & & 1 & 5 & 5 \\
\hline$t_{ \pm 1}$ & 1 & 1 & 1 & 1 & 1 & 1 & 1 & 1 \\
\hline$u_{ \pm 1} \ldots\{$ & 1 & 1 & & & 1 & 1 & 2 & 2 \\
\hline$v_{ \pm 1} \ldots\{$ & 1 & 2 & & & 2 & 1 & 1 & 1 \\
\hline$w_{ \pm 1} .$. & 2 & 3 & & & 3 & 2 & 1 & 1 \\
\hline
\end{tabular}

2. The Finiteness of ALGEBRAIC Systems

Consider now the following well-known lemma on systems of polynomials, first proved by Hilbert: If an infinite system of homogeneous forms in $n$ variables satisfies any law sufficiently explicit to locate an arbitrarily chosen homogeneous form within or without the system, then there always exists a set of these forms, finite in number, as $F_{1}, \cdots, F_{s}$, each within the system, 
and such that any form $F$ belonging to the system is expressible in the form

$$
F=T_{1} F_{1}+\cdots+T_{s} F_{s},
$$

where $T_{1}, \cdots, T_{8}$ are also homogeneous polynomials in the same $n$ variables but do not necessarily belong to the original system.*

The generality of this theorem with regard to the law or laws which determine the system of forms under consideration makes it applicable to a variety of problems, but the possibilities in connection with the study of particular given laws of formation and the systems appertaining have not been much exploited in literature.

When we apply the methods of this paper to systems of concomitants in the domain $R(1,0,0)$, functions which are free from the coefficients of the transformation $T$ and therefore are the ordinary rational integral concomitants of $f_{m}$, we discover a very interesting application of this lemma. The character of (1) as a typical representation of $f_{m}$ immediately shows that any concomitant $C$ of $f_{m}$ in $R(1,0,0)$ is a homogeneous polynomial $F$ in the invariants $\phi_{m-2 i}$ $(i=0, \cdots, m), f_{+1}, f_{-1}$, and formed according to a law sufficiently definite to define a system of polynomials in the technical sense of the lemma. This law is that $F$ shall be such a polynomial that, although its variables $\phi_{m-2 i}$, $f_{+1}, f_{-1}$ belong to the domain $R(1, T, \Delta)$, it simplifies by multiplying out into an expression (in $x_{1}, x_{2}, a_{0}, \cdots, a_{m}$ ) belonging to the domain $R(1,0,0)$, the parameters $\alpha_{1}, \alpha_{2}, \beta_{0}, \beta_{1}$ disappearing by cancellation from numerator and denominator.

Illustration. If $m=2$ we have; $\left(\gamma_{1}=\beta_{1}-\alpha_{1}\right)$,

$$
\begin{aligned}
& \phi_{ \pm 2}=\left(2^{3} \beta_{0}^{2} \Delta^{2}\right)^{-1}\left[\left(\gamma_{1}^{2}+2 \alpha_{2} \beta_{0} \mp \gamma_{1} \Delta\right) a_{0}+2 \beta_{0}\left(-\gamma_{1} \pm \Delta\right) a_{1}+2 \beta_{0}^{2} a_{2}\right], \\
& \phi_{0}=\left(2 \beta_{0} \Delta^{2}\right)^{-1}\left[\alpha_{2} a_{0}+\gamma_{1} a_{1}-\beta_{0} a_{2}\right],
\end{aligned}
$$

and

$$
4 \beta_{0}^{2} \Delta^{2}\left(\phi_{0}^{2}-4 \phi_{2} \phi_{-2}\right) \equiv a_{1}^{2}-a_{0} a_{2} \quad(=F) .
$$

We can now prove that the system of concomitants belonging to the domain $R(1,0,0)$ is finite (Gordan's theorem). For, suppose that $F$ is any such concomitant. It is a homogeneous polynomial in $\phi_{m-2 i}(i=0, \cdots, m)$, $f_{+1}, f_{-1}$, as above. Hence there exists a finite set of polynomials in $\phi_{m-2 i}$, $f_{+1}, f_{-1}$, as $F_{1}, \cdots, F_{s}$, each simplifying by multiplication into a concomitant belonging to the domain $R(1,0,0)$, such that

$$
F=T_{1} F_{1}+\cdots+T_{s} F_{s},
$$

and the polynomials $T_{i}$, being homogeneous in the invariants $\phi_{m-2 i}, f_{ \pm 1}$, are concomitants which may or may not belong to the domain $R(1,0,0)$.

* Mathematische Annalen, vol. 36 (1890), p. 473. 
We can now prove that the $T-s$ do, however, belong, in fact, to this domain. This will be equivalent to a proof of the finiteness theorem as each $T$ function can then be expressed linearly in terms of $F_{1}, \cdots, F_{s}$ and the process repeated until $F$ is reduced to a polynomial in the forms of the finite fundamental system $F_{1}, \cdots, F_{s}$.

The following propositions should now be noted: $(a)$ The selection of the set of concomitants $F_{i}$ in (10) is not unique. The expression of $F$ as a polynomial in $f_{+1}, f_{-1}, \phi_{m-2 i}(i=0, \cdots, m)$ is, on the other hand, unique, since we can obtain this expression (eq. (10)) by the substitution for $x_{1}, x_{2}, a_{0}$, $\cdots, a_{m}$ in $F$, the linear functions $\tau$ in $f_{1}, f_{-1}$, and the linear functions in $\phi_{m}, \cdots, \phi_{-m}$ obtained by solving for the inverse of equations (4). Note that the equations (4) are consistent for this solution. If the determinant of this linear system were zero, the $\phi_{m-2 i}$ would be linearly connected; then $a_{0}, \cdots, a_{m}$ would be linearly dependent. But these are arbitrary.

Although $F$ is a unique polynomial $P$ in $f_{1}, f_{-1}, \phi_{m}, \cdots, \phi_{-m}$ and (10) is merely a grouping of the terms of $P$ into components such that $F_{1}, \cdots, F_{8}$ are concomitants which simplify into the domain $R(1,0,0)$, nevertheless, even after the non-unique set $F_{1}, \cdots, F_{s}$ is definitely selected, the set of auxiliary factors $T_{1}, \cdots, T_{s}$ is not necessarily unique.*

(b) The linear transformation $T$ is generated by combination of the following particular transformations:

$$
\begin{aligned}
& T_{n}: x_{1}=\xi+n \eta, \quad x_{2}=\eta ; \\
& S_{k}: x_{1}=\xi, \quad x_{2}=k \eta(k \neq 0) ; \\
& V: x_{1}=-\eta, \quad x_{2}=\xi .
\end{aligned}
$$

Invariancy under $V$ is equivalent to the property of symmetry of invariants under the permutations $\left(a_{0} a_{m}\right)\left(a_{1} a_{m-1}\right) \cdots\left(x_{1} x_{2}\right)$. The transformation $S_{k}$ represents isobarism, and $T_{n}$, seminvariance. It follows that a homogeneous polynomial in $a_{0}, \cdots, a_{m}, x_{1}, x_{2}$ which possesses the properties of symmetry, isobarism, and seminvariance is a concomitant under $T$, and conversely.

(c) The equations of $r$, looked upon as linear transformations are as general as those of $T$.

(d) If $f$ is transformed into $f^{\prime}=\left(a_{0}^{\prime}, \cdots, a_{m}^{\prime} \backslash x_{1}, x_{2}\right)^{m}$ by a general linear transformation $t$ and if $I^{\prime}=I\left(a_{0}^{\prime}, \cdots, a_{m}^{\prime} ; x_{i}^{\prime}, x_{2}^{\prime}\right)$ is any homogeneous polynomial in its arguments which possesses the technical properties of symmetry, isobarism, and seminvariance, then $I^{\prime}$ is the left-hand member of the invariant relation of an invariant $I$, viz.,

$$
I\left(a_{0}^{\prime}, \cdots ; x_{1}^{\prime}, x_{2}^{\prime}\right)=D^{\wedge} I\left(a_{0}, \cdots ; x_{1}, x_{2}\right) .
$$

${ }^{*}$ Cf. some theory by the present writer, on expressing a polynomial in terms of others, in these Transactions, vol. 15 (1914), p. 77, and vol. 18 (1917), p. 443. 
To prove this, notice that the assumed properties of symmetry, isobarism, and seminvariance are sufficient conditions for the invariance of $I^{\prime}$ under the transformation of $f^{\prime}$ by a general linear transformation $t^{\prime}$. But if $f^{\prime}$ has a formal invariant $I^{\prime}$, under $t^{\prime}$, then, by correspondence, $f$ has a formal invariant $I\left(a_{0}, \cdots, a_{m} ; x_{1}, x_{2}\right)$ under $t$. Hence $I^{\prime}$ cannot be other than the transformed function of $I\left(a_{0}, \cdots, a_{m} ; x_{1}, x_{2}\right)$ under transformation of $f$ by $t$, which was to be proved.

(e) The concomitants $T_{i}$ belong to the domain $R(1, T, 0)$ if not to $R(1,0,0)$. For, if $f^{\prime}$ is the transformed of $f$ by $T$, the relation for $f^{\prime}$ analogous to $(10)$ is of the type

$$
(\alpha \beta)^{k} \cdot F=T_{1}^{\prime}(\alpha \beta)^{k_{1}} F_{1}+\cdots+T_{s}^{\prime}(\alpha \beta)^{k_{s}} F_{s} \quad[(\alpha \beta)=D],
$$

and this relation is necessarily identical with (10). Thus the invariant relation for $T_{i}$ is of the form

$$
T_{i}^{\prime}=(\alpha \beta)^{k_{i}^{\prime}} T_{i},
$$

where $k_{i}^{\prime}$ is a positive integer. Since the multiplier in this invariant relation involves $D$, and neither of its irrational factors $\rho_{1}, \rho_{-1}$, this amounts to a sufficient condition that $T_{i}$ belong to $R(1, T, 0)$.

Consider next the equation (1) of Section 1. This form $f^{\prime}=f_{m}$ is the result of transforming $f_{m}$ by a binary substitution $\tau$ of general type (cf. proposition $(c))$. We wish to show that the auxiliary factors $T_{1}, \cdots, T_{s}$ in (10) are functions $I^{\prime}$ of proposition $(d)$, i. e., they are functions of $\phi_{m-2 i}(i=0, \cdots$, $m), f_{+1}, f_{-1}$ which possess the formal properties of symmetry, isobarism, and seminvariance.

(A) Symmetry. That $T_{i}$ is unaltered, save possibly by sign, by the permutational substitution $S=\left(\phi_{m} \phi_{-m}\right)\left(\phi_{m-2} \phi_{-m+2}\right) \cdots\left(f_{1} f_{-1}\right)$ follows immediately from the fact that $T_{i}$ belongs to the domain $R(1, T, 0)$. For, it must be a linear function of combinations $P+Q$, or of $P-Q$, where $Q$ is a product of powers of $\phi_{m-2 i}(i=0, \cdots, m), f_{+1}, f_{-1}$ and $P$ is the product conjugate to $Q$. But the substitution $S$ interchanges $P$ and $Q$ (cf. (8)).

(B) Isobarism. The isobaric property of $T_{i}$ implies, in this instance, its invariance under the transformations

$$
\phi_{m-2 i}^{\prime}=k^{i} \phi_{m-2 i} ; \quad f_{+1}^{\prime}=f_{+1}, \quad f_{-1}^{\prime}=k^{-1} f_{-1}
$$

(cf. proposition (b)).

Now a representative term of $T_{i}$ is (cf. (5))

$$
P=\phi_{m}^{x_{0}} \phi_{m-2}^{x_{1}} \phi_{m-4}^{x_{2}} \cdots f_{+1}^{\sigma_{1}} f_{-1}^{\sigma_{2}},
$$

and both $\sigma_{1}+\sigma_{2}$ and $x_{0}+x_{1}+\cdots+x_{m}$ are invariable. If the transformations (12) are applied to $P$ the exponent of the power of $k$ which is a factor of the result is

$$
w=x_{1}+2 x_{2}+\cdots+m x_{m}-\sigma_{2} .
$$


But from equation (6),

$$
w=\frac{1}{2}\left[m\left(x_{0}+x_{1}+\cdots+x_{m}\right)-\left(\sigma_{1}+\sigma_{2}\right)\right],
$$

and this number is evidently invariable for the various terms $P$ of $T_{i}$. Hence the isobaric property is proved.

(C) Seminvariance. The seminvariance of $T_{i}$ is the property of remaining absolutely unaltered when we apply to it those substitutions upon $\phi_{m-2 i}$ $(i=0, \cdots, m), f_{+1}, f_{-1}$ which are induced by the transformations $T_{n}$ of proposition (b), viz.,

$$
f_{+1}=f_{+1}^{\prime}+n f_{-1}^{\prime}, \quad f_{-1}=f_{-1}^{\prime}
$$

applied to $f_{m}$ of (1).

Let us suppose now that $n$ is infinitesimal. Then, by a well-known bit of elementary analysis which need not be repeated here, the result of transforming $T_{i}$ by the induced transformations is

$$
\begin{aligned}
T_{i}^{\prime}=T_{i}+n\left[\phi_{m} \frac{\partial}{\partial \phi_{m-2}}+2 \phi_{m-2} \frac{\partial}{\partial \phi_{m-4}}\right. & +\cdots \\
& \left.+m \phi_{-m+2} \frac{\partial}{\partial \phi_{-m}}-f_{-1} \frac{\partial}{\partial f_{+1}}\right] T_{i} .
\end{aligned}
$$

Moreover, when the induced transformations are applied to both members of equation (10), $F$ and $F_{i}(i=1, \cdots, s)$ remain unaltered since these functions reduce to ordinary covariants in the domain $R(1,0,0)$. Then this result of transforming (10) takes the form

$$
F=T_{1}^{\prime} F_{1}+T_{2}^{\prime} F_{2}+\cdots+T_{s}^{\prime} F_{s} .
$$

Therefore, unless the quantity in brackets in (14) vanishes, this expression for $F$ is one of the other possible representations of $F$ as a linear function of $F_{i}(i=1, \cdots, s)$ constructed upon the principle that the set of auxiliary factors $T_{i}$ is not unique (cf. proposition $(a)$ ). But this is impossible by $(B)$, for, unless the bracket in (14) is zero, $T_{i}^{\prime}$ is not isobaric. Hence $T_{i}^{\prime}=T_{i}$ and the property of seminvariance is established.

Conclusion. Thus, by the principle of correspondence, in accordance with proposition $(d)$, we have established the relations

$$
\begin{array}{r}
T_{i}\left(\phi_{m}, \phi_{m-2}, \cdots ; f_{+1}, f_{-1}\right)=R^{\lambda} T_{i}\left(a_{0}, a_{1}, \cdots ; x_{1}, x_{2}\right) \\
\quad(i=1, \cdots, s),
\end{array}
$$

where $R$ is the determinant of $\tau$. We can now prove that $\lambda=0$, so that $T_{i}$ is absolutely equal to a concomitant belonging to the domain $R(1,0,0)$. This is done by showing that $T_{i}$ is of degree zero in the parameters $\alpha_{1}, \alpha_{2}$, $\beta_{0}, \beta_{1}$, a fact which is fairly evident from empirical considerations, but an analytic proof of which is not without interest. 
Note that the $T_{-s}$, though fractional in the parameters are homogeneous in them, and hence, by Euler's theorem,

$$
\nabla T_{i}=\left(\alpha_{1} \frac{\partial}{\partial \alpha_{1}}+\alpha_{2} \frac{\partial}{\partial \alpha_{2}}+\beta_{0} \frac{\partial}{\partial \beta_{0}}+\beta_{1} \frac{\partial}{\partial \beta_{1}}\right) T_{i}=t_{i}^{\prime} T_{i},
$$

where $t_{i}^{\prime}$ is the degree of $T_{i}$ in the parameters. Now suppose that

$$
t_{1}^{\prime}=t_{2}^{\prime}=\cdots=t_{r}^{\prime}
$$

and operate by $\nabla$ upon both members of (10). Since the $F-s$ are free from $\alpha_{1}$, $\alpha_{2}, \beta_{0}, \beta_{1}$, the result is

or

$$
t_{1}^{\prime}\left(T_{1} F_{1}+\cdots+T_{r} F_{r}\right)+\cdots+t_{s}^{\prime} F_{s}=0 ;
$$

$$
t_{1} P_{1}+t_{2} P_{2}+\cdots+T_{v} P_{v}=0,
$$

where the degrees $t_{1}, \cdots, t_{v}$ are all different and $v \geqq 1$. Next operate $v-1$ times in succession by $\nabla$ upon (16), and we get the linear system

$$
t_{1}^{k} P_{1}+t_{2}^{k} P_{2}+\cdots+t_{v}^{k} P_{v}=0 \quad(k=1, \cdots, v) .
$$

The determinant $C$ of this linear system is the circulant of the $v$ distinct numbers $t_{i}$, i. e.,

$$
C=\left|\begin{array}{cccc}
t_{1} & t_{2} & \cdots & t_{v} \\
t_{1}^{2} & t_{2}^{2} & \cdots & t_{v}^{2} \\
\cdot & \cdot & \cdot & \cdot \\
t_{1}^{v} & t_{2}^{v} & & t_{v}^{v}
\end{array}\right| \neq 0 .
$$

We would therefore have all $P_{-s}$ zero, and hence the contradiction $F=0$, against which eventuality there are only two alternatives, viz., first, that the $t-s$ should all be zero due to the fact that the numerator and denominator of each function $T_{i}$ are of the same degree in the parameters, or, secondly, that the $T_{-s}$ are free from the parameters. Thus $\lambda=0$, and (15) shows that the $T_{i}$ are concomitants belonging to $R(1,0,0)$. This completes the proof of the theorem of finiteness.

University of Pennsylvania 